Animals as a Therapeutic Modality in Psychiatry

Hannah G. Markin

English Department, Bowling Green State University

WRIT 1120: Research Writing

Instructor Klever

April 8, 2021 


\section{Animals as a Therapeutic Modality in Psychiatry}

Animal-Assisted Therapy (AAT) is the utilization of animals as a therapeutic strategy to help individuals cope with mental health conditions. They may participate in visitation programs or be used in goal-directed therapy sessions. The most common animals in AAT are cats, dogs, and horses, but rabbits, birds, llamas, and even dolphins have been known to participate.

Therapy animals are often used in counseling sessions, hospitals, and hospice care centers, but they can also visit patients at home as well. Unlike service or social support animals, therapy animals usually interact with several patients and are supervised by a trained therapist, psychologist, or handler. They can be broken down into three categories; therapeutic visitation animals, assisted therapy animals, and facility therapy animals. Therapeutic visitation animals are household pets that have been trained to interact with all types of people to relieve anxiety and create a comforting atmosphere. They can be found in rehab facilities, hospitals, workplaces, schools, airports, and other public areas. Assisted therapy animals work with psychologists or occupational therapists to aid individuals in reaching their mental goals. Facility therapy animals are animals that are held in a residential or therapeutic environment on a daily basis. Facility animals are qualified to work specifically with clients or residents for prolonged periods of time. An animal must be well-tempered, obedient, clean and well-groomed, current on all shots, courteous around other dogs and strangers, at least one year old, and well socialized in order to be approved as a therapy animal. They must also pass a test to determine their temperament and obedience level. The majority of therapy groups also require that the animal passes the American Kennel Club's Canine Good Citizen (CGC) test.

Animal-assisted therapy has been used to treat people with mental and physical illnesses for thousands of years. According to the first recorded use of AAT, horses were used to raise the spirits of severely ill individuals in Ancient Greece. Animals and humans were rehabilitated together in Belgium during the Middle Ages. Florence Nightingale discovered that small pets decrease stress and anxiety in both adult and adolescent psychiatric patients in the 
nineteenth century (Alliance..., 2018, para. 2-3). As a result, The York Retreat, a sanctuary for the homeless and mentally ill, started using animals in therapeutic environments. The American Red Cross and the Army Air Corps collaborated after WWII to create a program where injured and ill veterans could engage with and care for animals while recovering. The Human-Animal Bond theory was developed by Dr. Boris Levinson, a psychologist, and Konrad Lorenz, an Austrian Nobel Laureate in Physiology. This concept represented humans' natural need to bond with animals and nature in order to normalize the chaos of everyday life. Dr. Levinson began the first formal research. In 1961, he made an "accidental discovery" involving his dog, Jingles, while working with a young boy. He stepped away for a moment, leaving Jingles alone with the child, and when he returned, he discovered the boy playing with the dog. This inspired him to continue his experiments with Jingles and his young patients, through which he discovered that the presence of a dog during his sessions had a positive impact on impaired children. These patients were more content and likely to socialize with the dog than with people. Dr. Levinson later used the phrase "pet therapy" in reference to his dog's favorable effects on young patients in a therapeutic setting (Brady, 2017, para. 3-4). Animal-assisted therapy has been utilized for thousands of years and is effective in aiding in the treatment of mental conditions.

\section{Depression and Anxiety}

Anxiety disorders, which include generalized anxiety disorder, panic disorder, and social anxiety disorder, are the most common mental health condition among American adults, affecting 18.1 percent of the population per year. Mood disorders, including major depressive disorder, bipolar disorder, and seasonal affective disorder are the leading cause of disability. Furthermore, there is a high chance of developing depression with an anxiety disorder or vice versa. According to some researchers, sixty percent of people with anxiety will also suffer from symptoms of depression (Rodriguez, 2020, para. 1-2). Individuals suffering from anxiety with or without depression and contrariwise are encouraged to speak with a therapist to assist them in coping with these disorders' symptoms (Rodriguez, 2020, "Can Anxiety and Depression Be 
Treated Together?" section). However, it is often difficult to open up to another person about one's mental health struggles. Animal-assisted therapy can help those hesitant to speak with a therapist become more comfortable during their appointment and ultimately aid them in overcoming their symptoms. This, in combination with animals' effect on one's biochemistry, are what makes AAT so effective. Studies have shown that human-animal interaction "causes biochemical changes in the patient's brain and release[s] neurotransmitters that improve mood and cause relaxation and suppression of anxiety (Koukourikos et al., 2019, "Depression" section). These changes have shown to be effective in lessening symptoms of depression and anxiety.

Many studies investigating the influence of Human-Animal Interaction (HAI) on anxiety concentrate on individuals in healthcare environments such as hospitals who are susceptible to elevated levels of anxiety. The influence of a therapy dog's presence during routine dental cleaning was investigated in a pilot study conducted to assess the effect of dog-assisted therapy for individuals with a history of anxiety related to dental visits. The research included self-assessments of the level of anxiety perceived by the patient in prior dental encounters as well as the present experience with the help of a therapy dog to test the hypothesis. The results concluded that the therapy dog's presence significantly decreased the level of anxiety experienced by patients and lowered their blood pressure. Patients who received a therapy dog visit for twelve minutes had substantially lower systolic pulmonary artery pressure (the amount of pressure the blood exerts against the artery walls when the heart beats) during and after the intervention, as well as pulmonary capillary wedge pressure (an indirect estimate of left arterial pressure) during and after the intervention, relative to patients who received normal treatment and patients who received visits from a human volunteer, in a study of hospitalized patients with heart failure. Compared to the control groups, patients who received a dog therapy visit had the largest reduction from baseline in state anxiety sum score (Human..., 2020, "Child Health: \& 
Development: Mental Health" section). These are only two examples of hundreds of studies that prove the effectiveness of AAT in relation to anxiety.

An increasing number of studies indicate that animal-assisted therapy is efficacious as a complementary treatment for lessening symptoms of depression and improving mood. One study sought to determine how AAT affected the levels of depression in residents of a long-term care facility. The participants showed substantially lower depression levels, as determined by the Beck Depression Inventory, compared to the control group after being introduced to structured visits with a therapy dog for six weeks. In a study conducted with sixty-nine psychiatric inpatients, AAT resulted in a substantial increase in experiences with other patients over the span of four weeks in contrast to therapy without animals. Interactions included sociability, helpfulness toward others, activation, and responsiveness. According to the researchers, AAT can supplement the benefits of traditional therapy (Human..., 2020, "Child Health \& Development: Mental Health" section). Individuals with depression often struggle with social skills due to their mental illness. Contact with animals is shown to improve these skills, breaking down the barriers to their prospective marginalization and allowing them to engage with other people, which is critical for the recovery process. Working with animals can help with the mental deprivation caused by the disease and its symptoms, as well as increase the patient's quality of life. The tasks associated with the therapy animals encourage patients to be active and to practice routine self-care (Koukourikos et al., 2019, "Depression" section). Utilizing AAT to aid in the treatment of anxiety and depression has promising results and should continue to be researched and evaluated to further understand the full range of benefits that animals can provide.

\footnotetext{
ASD and ADHD

Attention-deficit/hyperactivity disorder (ADHD) and autism spectrum disorder (ASD) are both neurodevelopmental disorders, meaning it affects an individual's central nervous system. More than half of the people with ASD have symptoms of ADHD. In children with ASD, ADHD is
} 
the most common coexisting disorder. On the other hand, up to a quarter of children with ADHD show low-level symptoms of ASD, such as trouble with social skills or sensitivity to fabric textures, to name a few examples. With both disorders, brain development has been affected in some way, including the brain's executive functioning. Decision-making, impulse control, time management, concentration, and organizational skills are all governed by this system. Many children's social skills are affected as well (Children..., 2019, "About ADHD” section). AAT supports the development of coping mechanisms in children with ASD and ADHD.

Therapy animals can be used to help children with ADHD develop their memory retention through play and accountability. Improvements in memory and problem-solving abilities can help them improve their academic performance, which can lead to improved self-confidence. Additionally, walking and playing with a dog regularly decreases cortisol levels and raises oxytocin levels. This will benefit children with ADHD who experience tension and anxiety in the classroom (Culbert, 2019, para. 6-7). A study conducted in 2019 sought to determine if Canine-Assisted Interventions (CAI) helped children with ADHD manage their symptoms. The research evaluated children aged seven to nine years old who had not been medicated on medication previously and had ADHD. According to the authors, the children were divided into two groups and provided either traditional psychosocial interventions on their own or standard psychosocial interventions augmented with a trained therapy dog. According to the report, CAI was found to be successful in improving attention and social skills in children with ADHD. The traditional intervention produced similar outcomes, but it took twelve weeks for patients to note, while CAI took just eight weeks. One researcher stated the following; "In addition, the fact that parents of the children who were in the CAI group reported significantly fewer problem behaviors over time than those treated without therapy dogs is further evidence of the importance of this research." This study and many others have shown that AAT can be an effective complementary treatment with traditional therapy for children with ADHD. 
AAT to assist children on the autism spectrum is one of the most common instances and is beneficial to any child on the spectrum, regardless of where they land. When a nonverbal child is encouraged to read to a therapy dog rather than a speech therapist or reading coach, it is often more effective. Animals' ability to control hormone levels can also benefit children experiencing sensory stimulation or having a meltdown. The physical response of these children's bodies to animals will help them find ways to self-regulate during times of stress (Culbert, 2019, para. 8-9). In contrast to the low sensory response caused by ASD, animals yield powerful multi-sensory stimuli such as strong and distinct repeated sounds, vivid visual images, distinct and unique scent, and friendly touch. This relates to animals' ability to interact with them in a non-verbal manner. Animals are not only effective companions, but they also have opportunities for social contact and bonding by empowering children with ASD. At the same time, they assist the child in interacting with others and reducing their anxiety (Koukourikos et al., 2019, "Autism” section). One study compared two types of occupational therapy for children with autism: one using standard techniques and the other using therapy animals. In comparison to children in sessions using only standard occupational therapy techniques, the results suggest that children in animal-assisted sessions displayed substantially more use of language and engaged in significantly more social interaction (Human..., 2020, "Child Health \& Development: Autism Spectrum Disorder" section). Based on current research, it is clear that animal-assisted therapy has a positive and helpful effect on children with ASD and ADHD.

\section{Alzheimer's and Dementia}

One of the most common and debilitating diseases among the elderly is Alzheimer's Disease. More than three million Americans suffer from this disease and with the aging population increasing, these numbers will continue to rise. As brain cell connections and the cells themselves degenerate and die, patients lose their memory along with other major mental functions. There is no cure for this disease although it can be somewhat managed with 
medications. Many families of those affected are turning to animal-assisted therapy to make the most of what time they have left.

Animals, particularly dogs, have been involved in visiting programs for elderly dementia patients for many years, providing an opportunity to form affectionate and enjoyable bonds. It has been shown that animal-assisted therapy can improve the social skills of patients suffering from dementia. This is further proven with the story of James, a six-year-old English Springer Spaniel and certified therapy dog. James regularly visits Birmingham Green Nursing Home and Assisted Living in Manassas, Virginia. The facility has several dementia units. Patton states, "When we arrive, he's so excited and happy to be there. Then you see this special kind of calm and compassion come over his face." He knows to wait for certain residents to approach him first, and with the others, he'll wait to be asked to put his head on their lap (Shaw, 2007, para. 3). James seems to have an internal timer and knows when it is time to say goodbye. This is important as too much excitement can be overstimulating and distressing to dementia patients. James has several remarkable stories revolving around his interactions at Birmingham Green. (Shaw, 2007, para. 4). The pet therapists planned a skit one Christmas, in which they walked the dogs around to music. "There was a lady who had not spoken or shown any kind of interest in the world around her for a very long time," Terry Patton, James' owner says. "She actually got up, went over and laid down beside James, and then insisted on taking the leash. We'd go every couple of weeks, and there she'd be, waiting at the end of her bed for us to come in" (Shaw, 2007, para. 5). James, and other therapy dogs like him, have an extraordinary amount of success stories when it comes to comforting dementia patients, which further proves AAT's effectiveness in assisting with the treatment and coping of psychiatric conditions.

In addition to the social issues a patient with Alzheimer's faces, there are challenges including movement, severe anxiety and rage, and confusion. Animal-assisted therapy has shown to comfort patients during particularly difficult times known as the "West syndrome" when a patient suffers more intense feelings during early evening hours. "The presence of the 
treatment animal has a complacent action in the mental state of patient[s], providing security and companionship" (Koukourikos et al., 2019, "Dementia” section) Other concerns with this disease include nutrition, as patients will often forget to eat meals or eat food that is not nutritional thus causing severe weight loss, affecting brain function. Caretakers thinking outside the box have discovered other animals to assist with this issue: "One study of patients with Alzheimer's Disease found that after the introduction of an aquarium into the facility, patients experienced higher food intake and weight gain and a reduced requirement of nutritional supplements" (Human..., 2020, "Healthy Aging: Mental Health" section). Alzheimer's and dementia have several negative effects on the mental health of patients, but therapy animals can certainly help alleviate much of the loneliness and stress that comes with these diseases.

\section{Risks of AAT}

Animal-assisted therapy is a wonderful tool that can be used to aid in the treatment of an array of psychiatric conditions. However, there are risks associated with it, the most prominent being zoonotic diseases. Elderly, sick, and immunocompromised individuals are the most at-risk for becoming seriously ill. This chance for exposure increases in human hospitals. Even if the animals go through screening before entering care facilities, they could still be bringing zoonoses out from these facilities and into the community. Researchers at Tufts University released survey results of existing policies "related to animal health and behavioral prerequisites for therapy animals and Animal-assisted intervention...programs" (Halpern, 2017, para. 7). The participants included forty-five hospitals, forty-five elder care facilities, and twenty-seven therapy animal organizations in the United States. Researchers found that many programs had insufficient preventative guidelines to minimize the potential spread and exposure of zoonotic diseases from animals to humans (Halpern, 2017). It is important to understand the risks associated with any type of therapy or treatment, and the possible spread of diseases is an especially important possibility to consider. 
Of course, nothing is a perfect solution, and animal-assisted therapy is no exception. Zoonotic diseases are certainly something that should be taken seriously, as serious harm could come to those who are exposed. However, this should not prevent hospitals, care facilities, and individuals from seeking out and continuing AAT programs, as there are ways to significantly minimize the probability of humans becoming ill. Therapy animals should have health screenings completed by a veterinarian prior to registration to ensure they are free of transmissible disease and parasites and have all necessary vaccinations. If an animal appears ill, they should not be doing visitations until they are confirmed to be healthy. Grooming is also incredibly important. Animals visiting patients, especially those in hospitals, must be clean. Therapy animals should be bathed or cleaned no longer than twenty-four hours prior to a visit. Claws and hooves should also be trimmed to prevent injury. Special attention should be paid to animal waste, as it is a very common way of spreading disease. Any equipment used during the visit must be thoroughly sanitized and hand hygiene protocols must be followed (Pet Partners, 2020). It is important to do everything possible to prevent zoonotic diseases from spreading so that animal-assisted therapy remains a helpful and beneficial tool for those who need it.

\section{Conclusion}

Animal-assisted therapy is a fantastic way for individuals to cope with the myriad of symptoms that come with many psychiatric conditions. From excess worry to agitation, animals have proven to be a great way to ease the stress and loneliness that comes with mental health struggles. While AAT has been utilized for thousands of years, the research behind it is relatively new. It is important that this research continues in order for us to fully comprehend the benefits. Based on studies that have been completed, however, animal-assisted therapy shows promising results in terms of helping psychiatric patients to cope and connect with the world around them and should continue to be utilized. 


\section{References}

Children and Adults with Attention-Deficit/Hyperactivity Disorder (CHADD). (2019, March 6). ADHD and autism spectrum disorder. https://chadd.org/about-adhd/adhd-and-autism-spectrum-disorder/

Culbert, D. (2019, April 8). Animal therapy and learning disability - what is the relationship? Learning Disability Today. https://www.learningdisabilitytoday.co.uk/2019/animal-therapy-and-learning-disability-wh at-is-the-relationship

Halpern, N. E. (2017, July 18). Potential risks from pet therapy programs. Fox Rothschild. https://animallaw.foxrothschild.com/2017/07/22/potential-risks-from-pet-therapy-progra $\underline{\mathrm{ms} /}$

Kokosky, J. (2018, August 13). Children with ADHD may benefit from canine assisted interventions. Contemporary Clinic. https://www.contemporaryclinic.com/view/chil

Koukourikos, K., Georgopoulou, A., Kourkouta, L., \& Tsaloglidou, A. (2019). Benefits of animal assisted therapy in mental health. International Journal of Caring Sciences, 12(3), 1898-1905. http://internationaljournalofcaringsciences.org/docs/64 koukorikos review 12 3.pdf

Pet Partners. (2020, February 20). The importance of infection control during therapy animal visits.

https://petpartners.org/blog/the-importance-of-infection-control-during-therapy-animal-vi sits/

Rodriguez, D. (2020, September 29). How to cope with anxiety and depression. EverydayHealth.com. https://www.everydayhealth.com/anxiety/anxiety-and-depression.aspx 
Shaw, G. (2020, January 31). Pet therapy offers many benefits for people with dementias. Brain and Life Magazine.

https://www.brainandlife.org/articles/dementia-therapy-goes-to-the-dogs/

The Human Animal Bond Research Institute. (2020, March 5). https://habri.org/ 